\title{
Evaluation of Gram-negative bacterial infection by a stable and conjugative bioluminescence plasmid in a mouse model
}

\author{
Yao-Kuang Huang ${ }^{1,2}$, Chishih $\mathrm{Chu}^{3}$, Chih-Hsiung Wu', Chyi-Liang Chen ${ }^{4^{*}}$ and Cheng-Hsun Chiu ${ }^{1,4,5^{*}}$
}

\begin{abstract}
Background: The green fluorescence protein (GFP)-associated fluorescence method and the luciferase-associated bioluminescence method are the two major methods for IVIS imaging system to investigate the bacterial infection in animal models. The aim of this study was to evaluate the infection route of Gram-negative bacteria carrying a stable and broad range of conjugative bioluminescence plasmid PSE-Lux1 in a mouse model.
\end{abstract}

Results: Both encapsulated and non-encapsulated Gram-negative bacteria were used as hosts to evaluate conjugation efficiency and plasmid stability of pSE-Lux1, a recombinant of pSE34 and luXABCDE operon. The plasmid conjugation efficiencies of PSE-Lux1 ranged from $10^{-3}$ to $10^{-7}$ in various Gram-negative bacteria. Plasmid PSE-Lux 1 maintained in Escherichia coli, Klebsiella pneumoniae, and Salmonella enterica serovars Choleraesues (abbreviated S. Choleraesuis) and Typhimurium (S. Typhimurium), than in Acinetobacter baumannii and Serratia marcescens, was shown to be of better stability for at least four days. To investigate systemic bacterial infections, K. pneumoniae strain CG354 was intravenously injected, and then was clearly observed to be non-pathogenic to Balb/c mice for a long-term bioluminescence monitoring for 6 days. For examining dynamic distributions of gastrointestinal tract infection, the invasion protein SipB-deficient mutant OU5045 $\triangle$ sipB and OU5046 $\triangle \operatorname{sipB}$ of S. serovar Typhimurium constructed in this study, compared to wild-type strain OU5045 and its virulence plasmid-less strain OU5046, were of less virulence to mice.

Conclusions: This is the first study to evaluate the conjugative and stable bioluminescence vehicle system of pSE-Lux 1 in a wide range of Gram-negative bacteria, a system that can provide a useful reporter approach to trace systemic and gastrointestinal bacterial infections in a mouse model.

Keywords: Conjugative plasmi, Plasmid stability, Bioluminescence, Mutagenesis, Gram-negative bacteria

\section{Background}

Traditional in vivo animal models to investigate microbiologic infection require sacrifice for tissue smear and culture. Although numerous methods have been developed to image bacteria, the clinical applications are still limited due to inefficient in vivo detections for a long term of observation without additions of selective antibiotics and substrates, such as lucifirins [1]. Recently, bioluminescence expression system has been developed to

\footnotetext{
* Correspondence: dinoschen@adm.cgmh.org.tw; chchiu@adm.cgmh.org.tw ${ }^{4}$ Molecular Infectious Disease Research Center, Department of Medical Research, Chang Gung Memorial Hospital, No. 5, Fu-Hsin Street, Kweishan, Taoyuan, Taiwan

'Graduate Institute of Clinical Medicine, College of Medicine, Taipei Medical University, Taipei, Taiwan

Full list of author information is available at the end of the article
}

trace microorganisms in a living animal [2]. Bioluminescence system in live bacteria is regulated by a $\operatorname{lu} x A B C D E$ operon, in which $\operatorname{lux} A B$ genes encodes for luciferases and lux $C D E$ genes encode lipid acid reductases to catalyze the reduction of long chain lipid acids into aldehyde compounds that are the substrates specific for the catalysation of luciferases LuxA and LuxB [3].

Recently, many luxABCDE-carrying vectors have been constructed for bioluminescence assays; however, a steady, broad-host auto-bioluminescence vehicle is needed, especially a conjugatable plasmid is preferred to deliver exogenous DNA between bacteria and overcome the difficulty in transformation for some thick-capsule bacteria, such as Klebsiella pneumoniae [2,4]. Earlier, we constructed a 
conjugative vehicle pSE-Lux1, which is a chimeric vector in combination of a stable conjugative native pSE34 that include pilX1, pilX2, pilX4, pilX5, pilX6, pilX7, pilX8, pilX9, pilX10, pilX11, $\operatorname{tax} A, \operatorname{tax} B$, and $\operatorname{tax}$ of type IV secretion system, with a bioluminescence reporter p3ZLux4 that contains the luxABCDE-kan operon of pXen-5 (Bioware, Caliper Life Sciences, USA) and the portion of pGEM ${ }^{\circ}-3 Z$ (Promega Corporation, USA) with 300-400 copies per bacterial cell [5-8]. Although pSE34 carries pir, parGF and $s t b D E$ for plasmid stability and partition, plasmid stability of pSE34 has not been evaluated [7].

In this study, the bacterial conjugation efficiency and plasmid stability of pSE-Lux1 were determined in various Gram-negative bacteria, and distributions of the pSE-Lux1-carrying bacteria in Balb/c mice were evaluated by an in vivo imaging system.

\section{Methods}

\section{Bacterial strains}

The Gram-negative bacteria and plasmids used in this study are listed in Table 1. Bacteria were routinely incubated with Luria-Bertani (LB) media (Difco ${ }^{\mathrm{Tm}}$, Becton Dickinson, USA). Salmonella serovars were verified by the $\mathrm{O}$ - and $\mathrm{H}$-antigen agglutination tests $\left(\mathrm{Difco}^{\mathrm{rm}}\right)$. Plasmid was checked using the alkaline lysis method [9]. Antimicrobial agents ampicillin $(100 \mu \mathrm{l} / \mathrm{ml})$, chloramphenicol $(30 \mu \mathrm{l} / \mathrm{ml})$, gentamicin $(50 \mu \mathrm{l} /$ $\mathrm{ml})$, and kanamycin $(50 \mu \mathrm{l} / \mathrm{ml})$ were used for bacterial selection.

\section{Mutagenesis in S. Typhimurium}

Invasion effector gene sipB gene of Salmonella pathogenicity island 1 (SPI-1) was chosen to construct less virulence mutants of $S$. Typhimurium via the phage Lambda Red recombinase expression system $[12,13]$. The primer sequences were designed according to the sequences of $\operatorname{sip} B$ of S. Typhimurium LT2 (accession number NC_003197) and cat (a chloramphenicol acetyl transferase-encoding gene) of pKD3 [13]. The forward sequence SipB-Cm-F (5'TGGAGTCTCG TCTGGCGGTA TGGCAGGCGA TG ATTGAGTC cgcctacctg tgacggaaga-3') and the reverse sequence SipB-Cm-R (5'-GCTGCGGTAT TCGTGACTTC CATGCCCAAC GCCACTTTAT ccctgccact catcgcagta-3') were generated a 932-bp PCR amplicon, where the $\operatorname{sip} B$ gene portion is presented in capital letter and the cat portion in lower case. S. Typhimurium strains OU5045 and OU5046 had been previously transformed with a Lambda Red recombinases-carrying pKD46Gm by electroporation $[10,13]$. The $S$. Typhimurium $\operatorname{sip} B$-deleted mutants were named OU5045 $\triangle \operatorname{sip} B$ and OU5046 $\triangle \operatorname{sip} B$.

\section{Plasmid conjugation and stability}

The conjugation test was conducted with a slight modification of methods described elsewhere [7]. E. coli TOP10 and $S$. Typhimurium OU5046 carrying pSELux1 played as donors, the drug-susceptible recipient bacteria, including A. baumannii strains AB08 and AB23, E. coli TOP10, K. pneumoniae strain CG354, S. Typhimurium OU5045 and OU5046, and S. Choleraesuis OU7085,

Table $1 \mathrm{Gram}$-negative bacterial species and plasmids used in this study ${ }^{\#} \mathrm{Ap}^{\mathrm{r}}$ : ampicillin resistance; $\mathrm{Cm}^{\mathrm{r}}$ : chloramphenicol resistance; $\mathrm{Gm}^{\mathrm{r}}$ : gentamicin resistance; $\mathrm{Km}^{\mathrm{r}}$ : kanamycin resistance; Str $^{\mathrm{r}}$ : streptomycin resistance

\begin{tabular}{|c|c|c|}
\hline Strains and plasmids & Characteristics $^{\#}$ & Note \\
\hline E. coli TOP10 & $\Delta$ lacX74 aras $139 \Delta$ (ara-leu) & Invitrogen ${ }^{\circledast}$ \\
\hline \multicolumn{3}{|l|}{ Salmonella Typhimurium } \\
\hline OU5045 & Wild type with a virulence plasmid & Ou and Chu [10] \\
\hline OU5046 & A virulence plasmidless strains derived from OU5045 & Ou and Chu [10] \\
\hline OU $5045 \triangle \operatorname{sip} B$ & OU5045 with $\operatorname{sip} B$ deletion; $\mathrm{Cm}^{\mathrm{r}}$ & This study \\
\hline OU5046॰sipB & OU5046 with $\operatorname{sip} B$ deletion ; $\mathrm{Cm}^{\mathrm{r}}$ & This study \\
\hline Salmonella Choleraesuis OU7085 & Clinical isolate; drug-susceptible & Ou and Chu [10] \\
\hline \multicolumn{3}{|l|}{ Serratia marcescens } \\
\hline CB40 & Encapsulated clinical isolate; $\mathrm{Cm}^{\mathrm{r}}$ & This study \\
\hline CB47 & Encapsulated clinical isolate; $\mathrm{Cm}^{\mathrm{r}}$ & This study \\
\hline \multicolumn{3}{|l|}{ Acinetobacter baumannii } \\
\hline AB08 & Encapsulated clinical isolate; $\mathrm{Cm}^{\mathrm{r}}$ & This study \\
\hline AB23 & Encapsulated clinical isolate; $\mathrm{Cm}^{\mathrm{r}}$ & This study \\
\hline Klebsiella pneumoniae CG354 & Encapsulated clinical isolate; $\mathrm{Cm}^{\mathrm{r}}$ & This study \\
\hline \multicolumn{3}{|l|}{ Plasmids } \\
\hline p3ZLux4 & ColE1-typed replicon and lux operon; Ap'; Km; & This study \\
\hline pKD46Gm & A temperature-sensitive plasmid of $\lambda$ Red mutagenesis vector; $\mathrm{Gm}^{\mathrm{r}}$ & Doublet et al. [11] \\
\hline pSE-Lux1 & 46.3-kb conjugative plasmid with IncX- and ColE1-typed replicon and lux operon; Ap', $\mathrm{Km}^{\mathrm{r}}$; & Chen et al. [7] \\
\hline
\end{tabular}


and Serratia marcescens strains CB40 and CB47, were electroporated with gentamicin-resistant plasmid the pKD46Gm [11]. Overnight donor and recipient bacteria were 10-fold diluted with LB broth and then co-cultured at $37^{\circ} \mathrm{C}$ without agitation for $16 \mathrm{hrs}$. Transconjugants and recipient bacteria were counted by plating bacteria on LB agar containing appropriate antibiotics. The conjugation efficiency of each recipient bacterium was determined by dividing the number of transconjugants by the total of recipients.

Plasmid stability was determined by methods as described elsewhere [14]. Bacteria were cultured in LB broth medium without antibiotic and subcultured twice a day for a period of four days (D0-D4) in a 1000-fold dilution (approximate 80 generations in four-day period). The number of bacteria (CFU) was counted on agar media with or without kanamycin and ampicillin for both pSE-Lux1 and p3ZLux4 (Table 1) to select the bacteria with or without the plasmid. The plasmid stability was determined by the ratio of the bacterial survival number on selective media to the total bacterial number on non-selective media $[5,6]$.

\section{Evaluation of bacterial infection in mice}

Animal test of bacterial infection in Balb/c mice was approved by the Institutional Animal Care and Use Committee (CGU11-164) of Chang Gung University, Taoyuan, Taiwan. Overnight-grown bacteria were washed and resuspended with phosphate buffered saline (PBS) and then was used to challenge the mice via intravenous (IV) injection and oral administration $[15,16]$. The mice were anesthetized by gas anesthesia (3\% isoflurane), and then imaged by the in vivo imaging system IVIS 100 Series (Xenogen) in accordance with the guidelines of Guide for the Care and Use of Laboratory Animals [17]. After animal experiments or once illness appearances, mice were euthanized according to the previous guidelines.

For a long-term bioluminescence test of a systemic bacterial infection using the pSE-Lux1-carrying bacteria in mice, the mice $(n=3)$ were intravenously injected with $10^{7} \mathrm{CFU}$ of $K$. pneumoniae CG354 (a strain nonpathogenic to mice). The mice were tested for 6 days without selective pressures, and then were euthanized by breaking the neck while still anesthetized.

For the evaluation of gastrointestinal infection of Salmonella in mouse, the mice were orally gavaged with $10 \%$ sodium bicarbonate to neutralize stomach acid for 30 minutes prior to Salmonella challenge. The mice were separated into four groups ( $n=5$ per group) and each mouse were then orally gavaged with $10^{9}$ CFU $S$. Typhimurium. The strains were wild type OU5045, virulence plasmid-less OU5046, and sipB-deletion mutant strains $\mathrm{OU} 5045^{\triangle} \operatorname{sip} B$ and $\mathrm{OU} 5046^{\triangle} \operatorname{sip} B$. The mice were tested without selective pressures for four hours to strains OU5045 and OU5046, and for two days to mutants due to the restriction of mouse illness appearance thereafter. The anatomic organs of the tested mice were analyzed in parallel for IVIS imaging.

\section{Statistical analysis}

Pair-wise comparison in one-way ANOVA (ANalyses Of VAriance between groups) test was performed using the software program of Statistical Product And Service Solutions (SPPS 12.0), and followed by a Tukey's HSD (Honestly Significant Difference) test to determine the significance of difference between p3ZLux4 and pSELux1 in the tests of plasmid stabilities.

\section{Results}

\section{Plasmid pSE-Lux1-mediated conjugation tests between} Gram-negative bacteria

The conjugation results showed that plasmid pSE-Lux1 enabled to be transferred into all tested Gram-negative bacteria, even into the clinical mucoid (encapsulated) $A$. baumannii, K. pneumoniae, and S. marcescens (Table 2). The highest conjugation efficiencies of pSE-Lux1 in the donor $E$. coli TOP10 were $7.5 \pm 2.4 \times 10^{-3}$ and $1.2 \pm 0.4 \times$ $10^{-3}$ for $S$. Choleraesuis SC7085 and S. marcescens CB47. Additionally, conjugation efficiencies were observed higher in the same species than between species; for examples, $E$. coli-to- $E$. coli versus $E$. coli-to- $S$. Typhimurium $(1.8 \pm 1.6 \times$ $10^{-4}$ vs. $\left.1.0 \pm 0.8 \times 10^{-6}\right)$, and conversely, $S$. Typhimuriumto-S. Typhimurium versus $S$. Typhimurium-to-E. coli $\left(1.4 \pm 1.6 \times 10^{-3}\right.$ vs. $\left.2.2 \pm 1.1 \times 10^{-6}\right)$. Furthermore, conjugation efficiency was strain-dependent. Transfer of pSE-Lux1 differed between two recipient $S$. marcescens strains CB47 and CB40 with a respective value of $1.2 \pm$ $0.4 \times 10^{-3}$ and $5.9 \pm 5.1 \times 10^{-7}$ (Table 2$)$.

Table 2 Conjugation efficiency of pSE-Kux1 among various Gram-negative species

\begin{tabular}{lll}
\hline Donor bacteria & Recipient bacteria & $\begin{array}{l}\text { Conjugation } \\
\text { efficiency }\end{array}$ \\
\hline E. coli/pSE-Lux1 & A. baumannii AB08 & $1.2 \pm 0.6 \times 10^{-7}$ \\
& A. baumannii AB23 & $8.1 \pm 3.8 \times 10^{-6}$ \\
& E. coli TOP10 & $1.8 \pm 1.6 \times 10^{-4}$ \\
& K. pneumoniae CG354 & $1.9 \pm 1.3 \times 10^{-6}$ \\
& S. Choleraesuis SC7085 & $7.5 \pm 2.4 \times 10^{-3}$ \\
& S. Typhimurium OU5045 & $1.0 \pm 0.8 \times 10^{-6}$ \\
& Serratia marcescens CB40 & $5.9 \pm 5.1 \times 10^{-7}$ \\
& Serratia marcescens CB47 & $1.2 \pm 0.4 \times 10^{-3}$ \\
S. Typhimurium & E. coli TOP10 & $2.2 \pm 1.1 \times 10^{-6}$ \\
OU5046/pSE-Lux1 & K. pneumoniae CG354 & $8.4 \pm 4.6 \times 10^{-7}$ \\
& S. Typhimurium OU5045 & $1.4 \pm 1.6 \times 10^{-3}$ \\
\hline
\end{tabular}

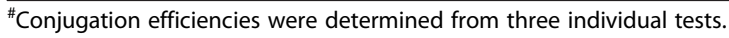


Difference in plasmid stability of pSE-Lux1 and p3ZLux4 within various bacteria

Plasmid stability of pSE-Lux1 and p3ZLux4 differed among non-capsulated $S$. Choleraesuis SC7085, S. Typhimurium OU4045 and OU5046, and E. coli TOP10 (Figure 1A). pSE-Lux1 maintained with at least $75 \%$ of stability at four-day duration (around 80 generations) in all four bacteria; the highest stability was observed in $S$. Choleraesuis SC7085 (almost 100\%). In contrast, p3ZLux4 was less stable $(<14 \%)$ than pSE-Lux1 in all tested bacteria with significant difference between these two plasmids $(P<0.001)$. Coincidently, p3ZLux 4 was the most stable in $S$. Choleraesuis SC7085 than other tested bacteria. In capsulated K. pneumoniae CG354, A. baumannii AB08 and $S$. marcescens, p3ZLux4 DNA was failed to transform into these bacteria by electroporation. Therefore, we only evaluated the stability of pSE-Lux1. The stability of pSE-Lux1 was higher in K. pneumoniae CG354 than in A. baumannii AB08 and S. marcescens CB47 (Figure 1B).
Long-term monitoring of bioluminescence bacterium in mice with a systemic bacterial infection

Non-pathogenic K. pneumoniae strain CG354 carrying pSE-Lux1 was used. The bioluminescence signals were clearly observed from the mice without selective pressures (Figure 2). Although the signal was gradually weakened within six-day period, the signal could still be clearly detected at the portion of mouse tail at the sixth day.

\section{Bacterial distributions of the route of gastrointestinal} infection in mice

Four S. Typhimurium isogenic strains OU5045, OU5046, and $\operatorname{sip} B$-deletion mutant strains $\mathrm{OU} 5045^{\triangle} \operatorname{sip} B$ and OU $5046^{\triangle} \operatorname{sip} B$ were transformed with pSE-Lux1. The bioluminescence signals from the mice demonstrated that most bioluminescence Salmonella travelled rapidly from stomach, small intestine, and large intestine to anal within four hours, and the bacteria were mostly shed at the $48^{\text {th }}$ hour post-bacterial inoculation (Figure 3).
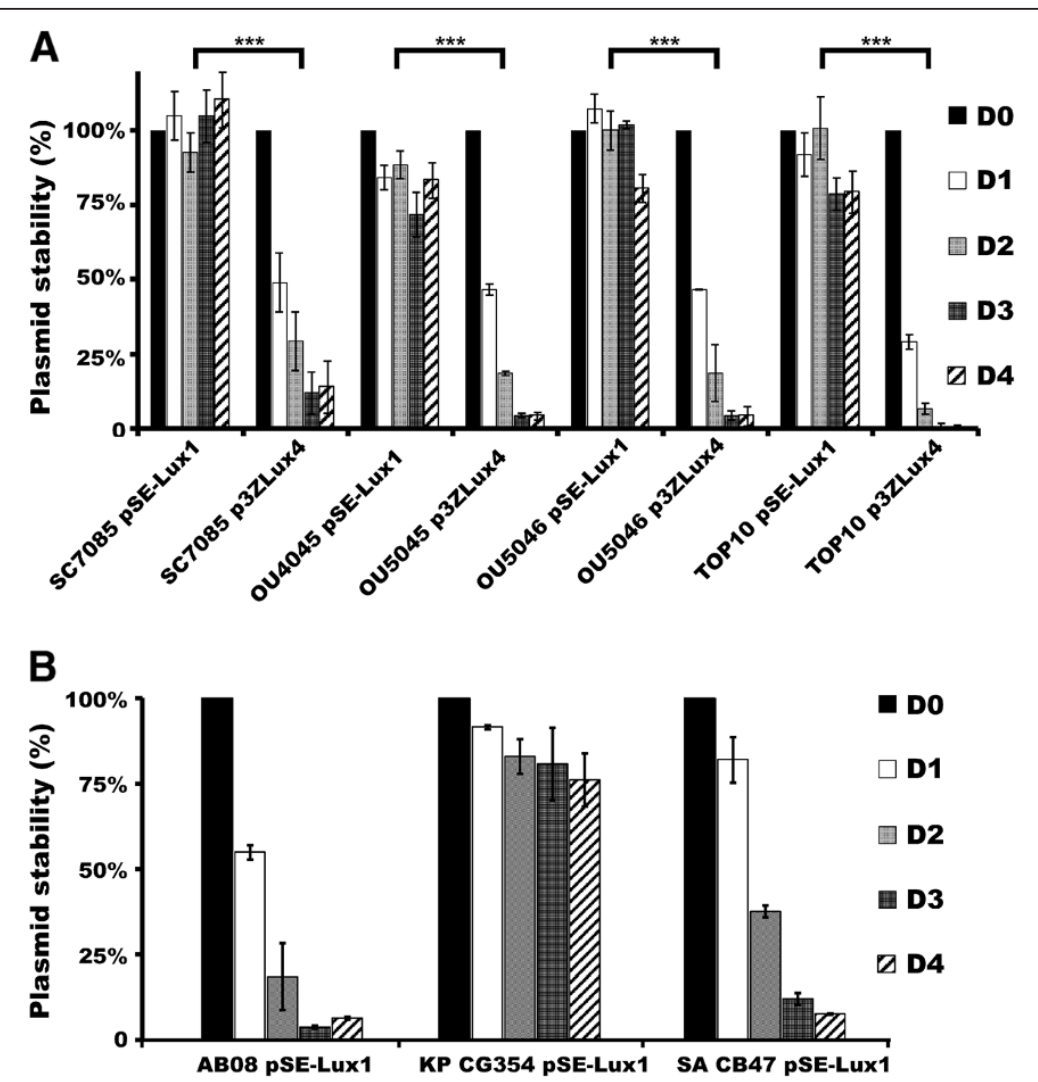

Figure 1 Plasmid stabilities. (A) The plasmid stabilities of pSE-Lux1 and p3ZLux4 are compared within various bacteria for four days. The tested bacteria include S. Choleraesuis SC7085, S. Typhimurium OU5045 and OU5046, and E. coli TOP10. ***: significance with $P$ value less than 0.001 $(P<0.001)$. (B) The plasmid stabilities of pSE-Lux1 within the encapsulated bacteria, including Acinetobacter baumannii $(A B) A B 08, K$. pneumoniae (KP) CG354, and Serratia marcescens (SA) CB47, were tested. D0 represents the day zero when the stability test was initiated, and its value was calibrated as 100\%, proportionally compared to those analyzed at the other time periods, including D1, D2, D3, and D4, which are denoted the day one, the day two, the day three, and the day four, respectively. 


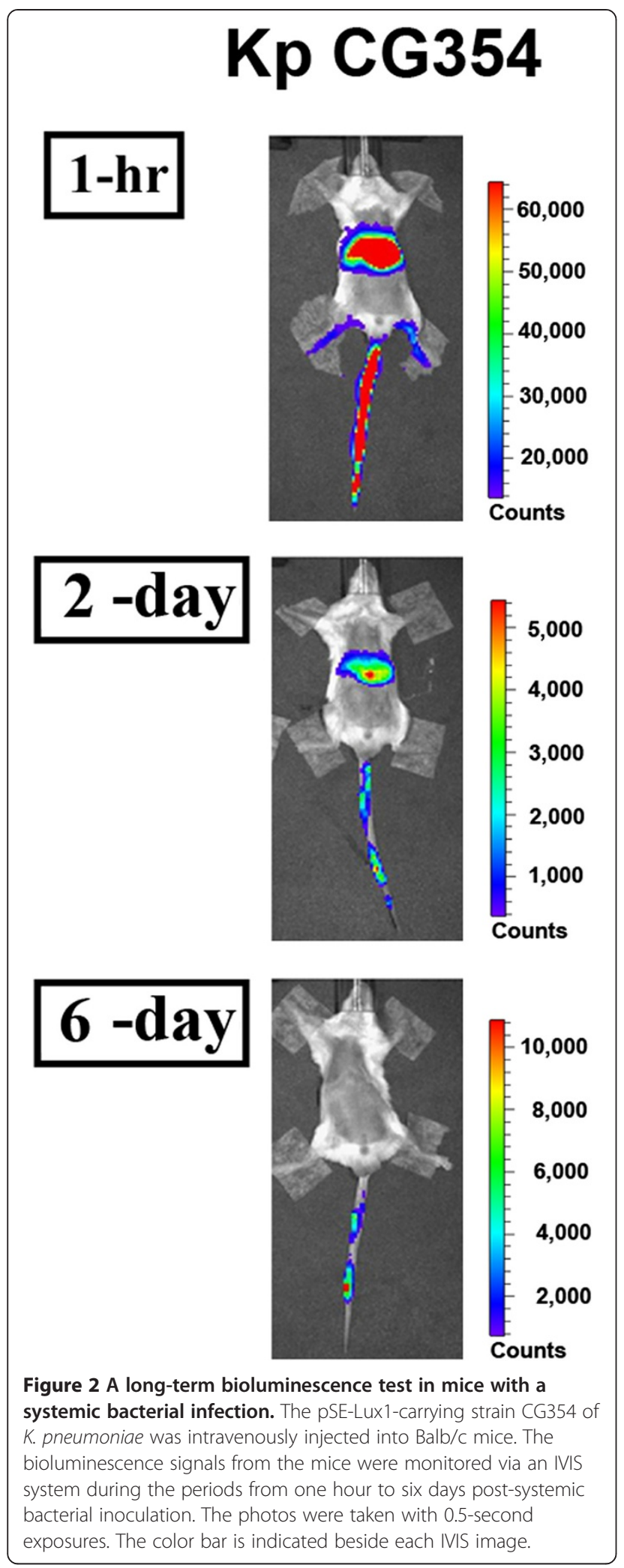

No significant difference in bioluminescence patterns was observed among the four isogenic strains within gastrointestinal tracts of mice. The bioluminescence signals were matched the amount of living bacteria from the anatomic organs. However, the periods of illness appearance differed between the $\operatorname{sip} B$-normal and the $\operatorname{sip} B$ deletion strains (four hours vs. two days post-Salmonella inoculations). Noteworthily, the bioluminescence signals present in the portion of stomach organs for the virulence plasmid-less OU5046, but not in its wild-type OU5045.

\section{Discussion}

The green fluorescence protein (GFP)-associated fluorescence method and the luciferase-associated bioluminescence method are the two major methods for IVIS imaging system in animal models. Compared to GFP fluorescence, two advantages of the $\operatorname{lux} A B C D E$-mediated bioluminescence method are A) only metabolically active and living bacteria may present light, but the dormant or dead bacteria may not, or weakly, because of their little production of aldehyde substrates for luciferase reactions; B) bioluminescence background of this system is low in animal models [18]. However, the GFP fluorescence method has higher sensitivity than bioluminescence methods in IVIS system and this advantage is only available to superficial organs less than $6 \mathrm{~mm}$ depth from the surface of test animals, or fluorescence signals would be faded [19]. Moreover, auto-fluorescence backgrounds emitted from animals are high [3]. To overcome auto-fluorescence, the test animals should get starved for 3 to 24 hours prior to fluorescence imaging, because diets may cause significant auto-fluorescence [20]. The precaution by starvation may limit the application of GFP-associated methods in IVIS imaging systems, particularly at the detection sites close to gastrointestinal tracts.

First bioluminescence image of the lux operon was developed to study the pathogenesis of $S$. Typhimurium in $\mathrm{C} 57 \mathrm{BL} / 6$ or BALB/c mice using an artificial plasmid that contains this operon from Photorhabdus luminescens [21]. However, the replication origin of this plasmid was derived from the ColE1 replicon and was unstable without antibiotic pressure. Therefore, three stable photonic plasmids pCGLS-1 (carrying ColE1 replicon), pAK1-lux (carrying pBBR1 replicon) and pXEN-1 (carrying both pC194 and ColE1 replicons) were constructed for the stability in $S$. Typhimurium [1]. In this study, a pSE34based pSE-Lux1 showed better plasmid stability than ColE1 replicon-based p3ZLux4 in E. coli, K. pneumoniae, and S. enterica. This is probably because pSE-Lux1 carries important genetic elements, including ColE1 and IncX replicons, conjugation-associated pil operon, and plasmid maintenance-associated genes pir, parG, parF, $s t b D$, and $s t b E$ [7]. Some other bioluminescence systems (such as fluc, gluc, or $r l u c$ ) may be more appropriate than $\operatorname{lu} x A B C D E$ in various bacteria $[2,3,22]$. However, firefly Fluc and Gaussia Gluc require the intravenous 

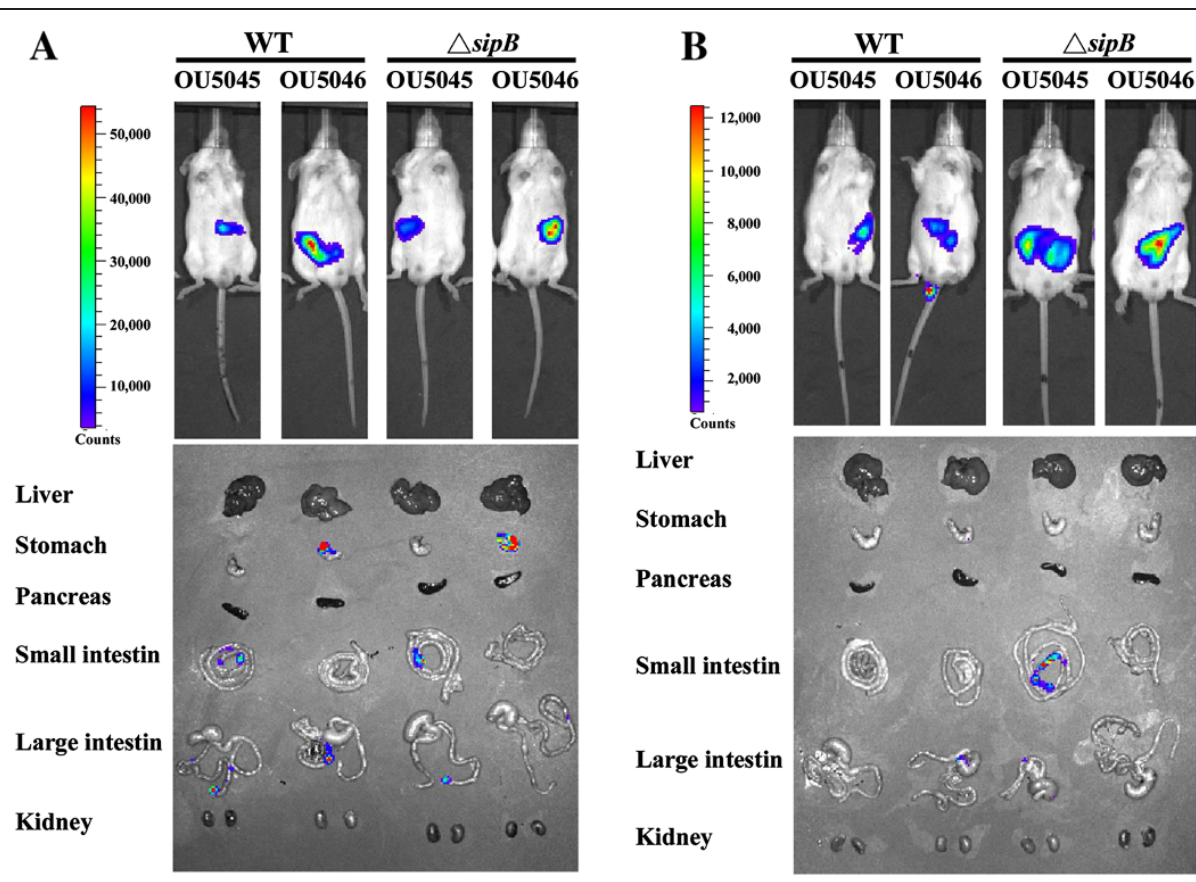

C
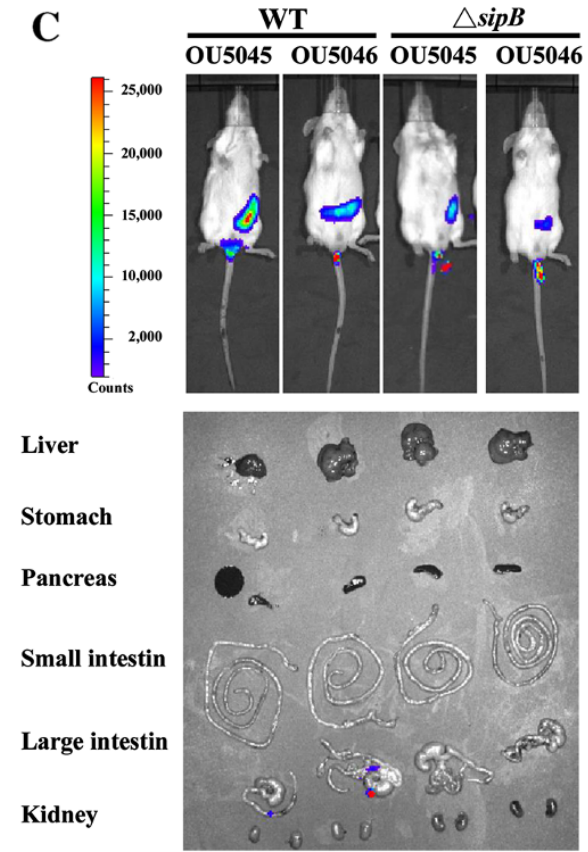

D
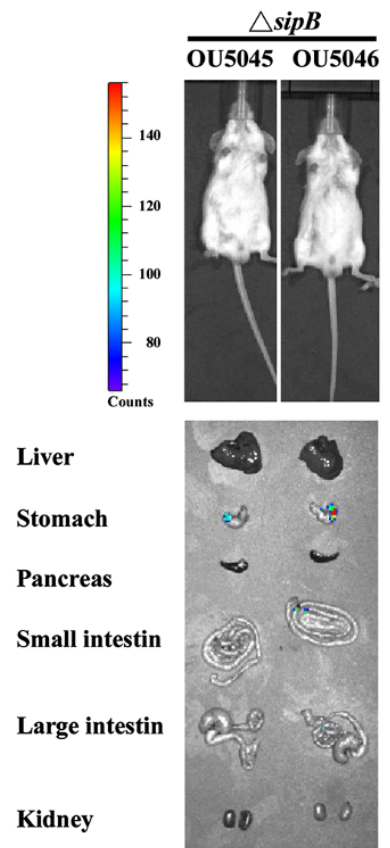

Figure 3 Dynamic distribution of Salmonella in mouse gastrointestinal tracts. The wild type (WT, including OU5045 and OU5046 strains) and sipB-deletion mutants (including OU5045 $\triangle$ sipB and OU5046 $\triangle$ sipB strains) of S. Typhimurium were orally administrated into Balb/c mice. The bioluminescence signals from living mice as well as from their anatomic organs in parallel (lower part of each panel) were monitored at the first- (A), second- (B), fourth- (C), and $48^{\text {th }}$-hour (D) periods after Salmonella inoculations. The pictures were taken with 3-minute exposures via IVIS. The color bar is indicated beside each IVIS image.

addition of substrate luciferins for bioluminescence catalyzation in vivo, therefore, it only remains relatively as short as 30 minutes when the peak of bioluminescence signal reaches a plateau [23].
The conjugation efficiencies differed between bacterial species probably due to difference in enzymatic restriction and modification system. However, the two recipient S. marcescens strain CB40 and CB47 showed 
dramatically different conjugation efficiencies and this difference may be attributed to the different genomic background or bacterial capsule. With regard to the bioluminescence patterns of $K$. pneumoniae mucoid strain CG354 for a systemic bacterial infection in this study, the gradual reduction of bioluminescence signals over the time indicated that strain CG354 was nonpathogenic to mice. Similar to strain CG354, strain IA565 of $K$. pneumoniae, a human clinical isolate, is known to be non-pathogenic to mice [24]. However, survey of International Klebsiella Study Group reported that $69 \%$ mucoid clinical strains are pathogenic to murine [16].

In the bioluminescence patterns of anatomic gastrointestinal organs of mice, pSE-Lux1-carrying OU5046 strain produced more signals in the stomach than by the pSE-Lux1-carrying OU5045 strain were found. It is likely because the more virulent strain caused more severe inflammatory diarrhea to mice, more Salmonella shed away from stomach and other gastrointestinal organs, and therefore, the less virulent $\triangle \operatorname{sip} B$ mutant remained more in gastrointestinal tracts. Although Salmonella are known to enable survive in the acidic environment of stomach through the induction of the acid tolerance response, it still remains unclear why Salmonella can colonize in the stomach; however, its colonization may explain why Salmonella can cause stomach cramps in humans $[25,26]$.

Bioluminescence-related publications have been increasing in application to study in the area of pathogenicity, tumorigenicity, biofilm, and dermatology [27-30]. Moreover, the bioluminescence vehicle can be genetically engineered to carry some other potential exogenous genes, such as anticancer agents for therapeutic purposes [31].

\section{Conclusion}

In this study, a novel stable and conjugative bioluminescence pSE-Lux1 vehicle system available in a broad range of bacteria, even for encapsulated bacteria, is well developed and applied to investigate the infection route of pSE-Lux1-carrying bacteria in living mice

\section{Competing interest}

No conflict of interest declared.

\section{Authors' contribution}

Study design and data collection: HY-K, Chen C-L, WC-H and Chiu C-H carried out the study design; HY-K, Chen C-L and Chiu C-H carried out the molecular data analysis. Chen C-L and Chiu C.-H carried out the experimental data interpretation. $\mathrm{HY}-\mathrm{K}$ and $\mathrm{Chen} \mathrm{C}-\mathrm{L}$ participated in the sequence alignment and drafted the manuscript. Chu C, Chen C-L and Chiu C-H refined the manuscript. All authors read and approved the final manuscript.

\section{Acknowledgements}

The authors thank Pei-Chun Tu and Hsin-Ju Chang, Chang Gung Memorial Hospital for their assistance in bacterial conjugation test and the IVIS detection in mice. This study was supported by grants from Chang Gung
Memorial Hospital, Taoyuan, Taiwan (CMRPG381051-2, CMRPG390701-2, CMRPG6C0341, CMRPG6B0501, CMRPG490141-3 and CMRPG3A1111-3).

\section{Author details}

${ }^{1}$ Graduate Institute of Clinical Medicine, College of Medicine, Taipei Medical University, Taipei, Taiwan. ${ }^{2}$ Division of Thoracic and Cardiovascular Surgery, Chang Gung Memorial Hospital, Chiayi, Taiwan. ${ }^{3}$ Department of Microbiology, Immunology, and Biopharmaceutics, National Chiayi University, Chiayi, Taiwan. ${ }^{4}$ Molecular Infectious Disease Research Center, Department of Medical Research, Chang Gung Memorial Hospital, No. 5, Fu-Hsin Street, Kweishan, Taoyuan, Taiwan. ${ }^{5}$ Division of Pediatric Infectious Diseases, Department of Pediatrics, Chang Gung Children's Hospital, and College of Medicine, Chang Gung University, No. 5, Fu-Hsin Street, Kweishan, Taoyuan, Taiwan.

Received: 17 June 2014 Accepted: 11 August 2014

Published: 19 August 2014

\section{References}

1. Moulton K, Ryan P, Lay D, Willard S: Photonic plasmid stability of transformed Salmonella typhimurium: a comparison of three unique plasmids. BMC Microbiol 2009, 9:152.

2. Andreu N, Zelmer A, Wiles S: Noninvasive biophotonic imaging for studies of infectious disease. FEMS Microbiol Rev 2010, 35:360-394.

3. Hutchens M, Luker GD: Applications of bioluminescence imaging to the study of infectious diseases. Cell Microbiol 2007, 9:2315-2322.

4. Llosa M, de la Cruz F: Bacterial conjugation: a potential tool for genomic engineering. Res Microbiol 2005, 156:1-6.

5. Chiu CH, Chen $\mathrm{CL}$, Huang YK, inventor: Chang Gung Memorial Hospital, assignee: Method for tracing Gram-negative bacteria inside animal model using stable and bioluminescence-based expression system therefor. In United States Patent. 2010:US8268616B2.

6. Chiu $\mathrm{CH}$, Chen $\mathrm{CL}$, Huang YK, inventor: Chang Gung Memorial Hospital, assignee: Method for tracing Gram-negative bacteria inside animal model using stable and bioluminescence-based expression system therefor. In 2011:US8263366B2.

7. Chen $\mathrm{CL}$, Wang CY, Chu C, Su LH, Chiu CH: Functional and molecular characterization of pSE34 encoding a type IV secretion system in Salmonella enterica serotype Enteritidis phage type 34. FEMS Immunol Med Microbiol 2009, 57:274-283.

8. Vuong C, Kocianova S, Yu J, Kadurugamuwa JL, Otto M: Development of real-time in vivo imaging of device-related Staphylococcus epidermidis infection in mice and influence of animal immune status on susceptibility to infection. J Infect Dis 2008, 198:258-261.

9. Kado Cl, Liu ST: Rapid procedure for detection and isolation of large and small plasmids. J Bacteriol 1981, 145:1365-1373.

10. Ou JT, Chu C, inventor: Crystal Biotechnology Research and Development Co., Ltd., assignee. In Chicken leucocytozoon vaccine. United States Patent. 1999:6207167.

11. Doublet B, Douard G, Targant H, Meunier D, Madec JY, Cloeckaert A Antibiotic marker modifications of lambda Red and FLP helper plasmids, pKD46 and pCP20, for inactivation of chromosomal genes using PCR products in multidrug-resistant strains. J Microbiol Methods 2008, 75:359-361.

12. Myeni SK, Wang L, Zhou D: SipB-SipC complex is essential for translocon formation. PloS One 2013, 8:e60499.

13. Uzzau S, Figueroa-Bossi N, Rubino S, Bossi L: Epitope tagging of chromosomal genes in Salmonella. Proc Natl Acad Sci U S A 2001, 98:15264-15269.

14. Trevors JT, van Elsas JD, Starodub ME, van Overbeek LS: Survival of and plasmid stability in Pseudomonas and Klebsiella spp. introduced into agricultural drainage water. Can J Microbiol 1989, 35:675-680.

15. Olivier V, Queen J, Satchell KJF: Successful small intestine colonization of adult mice by Vibrio cholerae requires ketamine anesthesia and accessory toxins. PLOS One 2009, 4:e7352.

16. Yu VL, Hansen DS, Ko WC, Sagnimeni A, Klugman KP, von Gottberg A: Virulence characteristics of Klebsiella and clinical manifestations of $K$. pneumoniae bloodstream infections. Emerg Infect Dis 2007, 13:986-993.

17. Kastenmayer RJ, Moore RM, Bright AL, Torres-Cruz R, Elkins WR: Select agent and toxin regulations: beyond the eighth edition of the Guide for the 
Care and Use of Laboratory Animals. J Am Assoc Lab Anim Sci 2012, 51:333-338.

18. Gahan CGM: The bacterial lux reporter system: applications in bacterial localisation studies. Curr Gene Ther 2012, 12:12-19.

19. Zacharakis G, Kambara H, Shih H, Ripoll J, Grimm J, Saeki Y: Volumetric tomography of fluorescent proteins through small animals in vivo. Proc Natl Acad Sci U S A 2005, 102:18252-18257.

20. Inoue Y, Izawa K, Kiryu S, Tojo A, Ohtomo K: Diet and abdominal autofluorescence detected by in vivo fluorescence imaging of living mice. Mol Imaging 2008, 7:21-27.

21. Contag CH, Contag PR, Mullins JI, Spilman SD, Stevenson DK, Benaron DA: Photonic detection of bacterial pathogens in living hosts. Mol Microbiol 1995, 18:593-603.

22. Tannous BA, Kim DE, Fernandez JL, Weissleder R, Breakefield XO: Codonoptimized Gaussia luciferase cDNA for mammalian gene expression in culture and in vivo. Mol Ther 2005, 11:435-443.

23. Chang MH, Cirillo SLG, Cirillo JD: Using luciferase to image bacterial infections in mice. J Vis Exp 2011, 18:2547.

24. Lau HY, Clegg S, Moore TA: Identification of Klebsiella pneumoniae genes uniquely expressed in a strain virulent using a murine model of bacterial pneumonia. Microb Pathog 2007, 42:148-155.

25. Alvarez-Ordóñez A, Begley M, Prieto M, Messens W, López M, Bernardo A: Salmonella spp. survival strategies within the host gastrointestinal tract. Microbiology 2011, 157(Pt 12):3268-3281.

26. Gonose T, Smith AM, Keddy KH, Sooka A, Howell V, Jacobs CA: Human infections due to Salmonella Blockley, a rare serotype in South Africa: a case report. BMC Res Notes 2012, 5:562.

27. Garcez AS, Núñez SC, Azambuja N, Fregnani ER, Rodriquez HMH, Hamblin MR: Effects of Photodynamic Therapy on Gram-Positive and GramNegative Bacterial Biofilms by Bioluminescence Imaging and Scanning Electron Microscopic Analysis. Photomed Laser Surg 2013, 31:519-525.

28. Gonzalez RJ, Weening EH, Frothingham R, Sempowski GD, Miller VL: Bioluminescence imaging to track bacterial dissemination of Yersinia pestis using different routes of infection in mice. BMC Microbiol 2012, 12:147.

29. lochmann S, Lerondel S, Bléchet C, Lavergne M, Pesnel S, Sobilo J: Monitoring of tumour progression using bioluminescence imaging and computed tomography scanning in a nude mouse orthotopic model of human small cell lung cancer. Lung Cancer 2012, 77:70-76.

30. Vecchio D, Dai T, Huang L, Fantetti L, Roncucci G, Hamblin MR: Antimicrobial photodynamic therapy with RLP068 kills methicillinresistant Staphylococcus aureus and improves wound healing in a mouse model of infected skin abrasion PDT with RLP068/Cl in infected mouse skin abrasion. J Biophotonics 2013, 6:733-742.

31. Forbes NS: Engineering the perfect (bacterial) cancer therapy. Nat Rev Cancer 2010, 10:785-794.

doi:10.1186/s12929-014-0078-y

Cite this article as: Huang et al:: Evaluation of Gram-negative bacterial infection by a stable and conjugative bioluminescence plasmid in a mouse model. Journal of Biomedical Science 2014 21:78.

\section{Submit your next manuscript to BioMed Central and take full advantage of:}

- Convenient online submission

- Thorough peer review

- No space constraints or color figure charges

- Immediate publication on acceptance

- Inclusion in PubMed, CAS, Scopus and Google Scholar

- Research which is freely available for redistribution

Submit your manuscript at www.biomedcentral.com/submit
Ciomed Central 